\title{
Augmented marker tracking for peri-acetabular osteotomy surgery
}

\author{
Silvio Pflugi ${ }^{1}$ (1) $\cdot$ Rakesh Vasireddy ${ }^{1} \cdot$ Till Lerch $^{2} \cdot$ Timo M. Ecker $^{2} \cdot$ Moritz Tannast $^{2} \cdot$ Nane Boemke $^{3}$. \\ Klaus Siebenrock ${ }^{2}$. Guoyan Zheng ${ }^{1}$
}

Received: 1 June 2017 / Accepted: 20 November 2017

(c) CARS 2017

\begin{abstract}
Objective To develop a hybrid augmented marker-based navigation system for acetabular reorientation during peri-acetabular osteotomy (PAO).

Methods The system consists of a tracking unit attached to the patient's pelvis, augmented marker attached to the acetabular fragment and a host computer to do all the computations and visualization. The augmented marker is comprised of an external planar Aruco marker facing toward the tracking unit and an internal inertial measurement unit (IMU) to measure its orientation. The orientation output from the IMU is sent to the host computer. The tracking unit streams a live video of the augmented marker to the host computer, where the planar marker is detected and its pose is estimated. A Kalman filter-based sensor fusion combines the output from marker tracking and the IMU. We validated the proposed system using a plastic bone study and a cadaver study. Every time, we compared the inclination and anteversion values measured by the proposed system to those from a previously developed optical tracking-based navigation system.

Results Mean absolute differences for inclination and anteversion were $1.34( \pm 1.50)$ and $1.21( \pm 1.07)^{\circ}$, respectively, for the cadaver study. Mean absolute differences were $1.63( \pm 1.48)$ and $1.55( \pm 1.49)^{\circ}$ for inclination and anteversion for the plastic bone study. In both validation studies, very strong correlations were observed.
\end{abstract}

Conclusion We successfully demonstrated the feasibility of our system to measure the acetabular orientation during PAO.

Keywords Computer-assisted surgery $\cdot$ Navigation $\cdot$ PAO surgery

\section{Introduction}

Peri-acetabular osteotomy (PAO) surgery is commonly carried out for the treatment of developmental dysplasia of the hip and is used to change the abnormal anatomy of the hip [52]. The abnormal anatomy and decreased area of the lunate surface in hip dysplasia result in axial overloading with decreased contact area, increased contact pressure and maximum overloading at the acetabular rim $[15,49]$. The goal of PAO surgery is to prevent or delay hip osteoarthritis in young and active patients [49]. It is a demanding, open surgery

Silvio Pflugi

silvio.pflugi@istb.unibe.ch

Guoyan Zheng

guoyan.zheng@istb.unibe.ch

1 Institute for Surgical Technology and Biomechanics, University of Bern, Bern, Switzerland

2 Department of Orthopedic Surgery, Inselspital, University of Bern, Bern, Switzerland

3 Institute for Anatomy, University of Bern, Bern, Switzerland where the surgeon separates the acetabulum from the rest of the pelvic bone using four cuts (osteotomies) $[7,16]$. PAO has the goal to correct and reorient the dysplastic acetabulum to increase femoral head coverage while maintaining joint stability $[16,52]$. It is important that the acetabular reorientation is performed accurately as acetabular malpositioning can lead to femoro-acetabular impingement (FAI) $[1,35]$ and decreased long-term outcome after PAO. Although several studies confirmed a favorable long-term follow-up $[29,50]$, the proper acetabular reorientation remains challenging. Computer-assisted surgery (CAS) techniques were proposed to improve the accuracy of orthopedic interventions $[19,26,39,47]$. Several CAS systems were proposed specifically for PAO [16,18,27,31,33,34]. Langlotz et al. [27] and Jäger et al. [18] presented a computed-tomography (CT)-based PAO navigation system for PAO. Liu et al. [31] proposed a computer-assisted planning and navigation system for PAO surgery including range of motion estimation. Murphy et al. [33,34] were the first to propose a system providing geometric and biomechanical parameters intra- 
operatively. They validated their system using a cadaveric and a clinical study, comparing their results to postoperative CT measurements as well as manual intra-operative measurements [33]. These navigation systems are all optical tracking-based navigation systems that use a relatively large stereo camera setup and need a constant line-of-sight (LOS) to the surgical instruments and the patient. Other tracking technologies were proposed to overcome the LOS impediment such as electromagnetic (EM) tracking $[25,54]$. EMbased navigation systems generally provide lower accuracy than optical tracking-based systems due to their susceptibility to magnetic field distortions. Other approaches for surgical navigation make use of inertial measurement units (IMUs) [2,40,42,45,46,53].

IMUs generally comprise a triaxial gyroscope that measures angular rate, a triaxial accelerometer which measures linear acceleration without gravity and a triaxial magnetometer which senses the surrounding magnetic field. Generally, IMUs use the accelerometer to define the "down" vector, the magnetometer to define the heading angle and the gyroscopes to drive the system dynamics that provides a prediction of the orientation. Drawbacks of IMUs include the accumulation of drift error due to the integration of the gyroscope and susceptibility to magnetic field distortions in case a magnetometer is used [2,40,46]. Sensor fusion (e.g., Kalman filter [22]) is needed to compute a final orientation estimate by combining the signals from each sensor included in the unit.

IMUs or individual sensors such as accelerometers recently became popular in orthopaedics. Commercial products such as KneeAlign $2^{T M}$ (OrthAlign Inc., USA) or iASSIST ${ }^{T M}$ (Zimmer Biomet, USA) are devices that make use of accelerometers for navigated total knee arthroplasty, and they have been heavily validated in clinical studies $[4,8,11-13$, 17,36-38]. Several studies suggest that accelerometer-based navigation is as accurate as imageless computer-assisted surgery systems for TKA $[8,17,38]$. Jost et al. [20,21] validated an IMU-based system [53] to assist with the implantation of pedicle screws and reported acceptable accuracy in the range of $0-5^{\circ}$. IMUs are not just used for navigation but can also be used for patient registration, especially for acetabular cup placement or PAO surgery. Cao et al. [5], similar to our previous work [42], use an IMU to measure the orientation of the anterior pelvic plane. To estimate the acetabular cup or fragment orientation, no translation is needed; therefore, IMUs are a reasonable alternative to large tracking setups. Cao et al. [5] validated their system using plastic bones and reported pose estimation errors of less than $1.2^{\circ}$. This error of course does not include the error of unknown soft tissue between the bony landmarks and the measurement device.

Another approach is to use planar markers known from augmented reality for the tracking of surgical instruments $[23,43]$. Planar markers are attached to the patient and sur- gical instruments and are tracked by a monocular camera attached to the operating room (OR) lamp [23] or the patient itself [43]. Planar marker tracking accuracy is depending on several things such as camera resolution, distance to camera, marker size and lighting conditions. One drawback of using planar markers is the possibility of blood splatters that may cover the marker, depending on the location of the marker. This however, is also the case with active or passive spherical markers used with optical tracking. Hybrid navigation systems have been proposed, combining two or more tracking technologies in a way to overcome each individual tracking technology's drawbacks [3,6,14,32]. Su et al. [51] combine a monocular camera with an IMU for prosthesis pose estimation during total hip replacement surgery. The camera and the IMU are mounted inside the femoral head prosthesis, and a customized pattern is printed on the internal surface of the acetabular cup. The reported relative error is less than $4.8 \%$ when data from the IMU are fused with the pose estimation from the camera. However, they only validated their system using plastic bones.

In this work, we propose to combine planar marker tracking with an IMU into a small and easy to use hybrid navigation system for PAO. The system consists of a tracking unit with a monocular camera directly placed on the patient's pelvis and an augmented marker that is placed on the patient's acetabular fragment (see Fig. 1). The augmented marker combines a planar Aruco marker [10] with a lowcost IMU. The augmented marker with the IMU allows a more convenient patient registration approach and makes it possible to overcome periods when the planar marker is not visible by the tracking unit. The contribution of this work is the removal of the LOS impediment by fusing the information from planar marker tracking and the IMU. Additionally we present a convenient patient registration method based on measuring the orientation of the anterior pelvic plane. We demonstrate the feasibility of the proposed system by using it to track the acetabular fragment orientation during PAO surgery. The system, however, could potentially be used for other types of orthopedic surgeries as well.

The paper is divided into two parts: the first part introduces the system including marker tracking, calibration, patient registration and sensor fusion. The second part covers validation of the system using a cadaver study (without sensor fusion) and a plastic bone study (including sensor fusion).

\section{Material and methods}

\section{System overview}

The system comprises of three parts: 1) The tracking unit which consists of a miniature computer (Raspberry Pi Zero, Raspberry Pi Foundation, UK), a WiFi USB dongle and a 

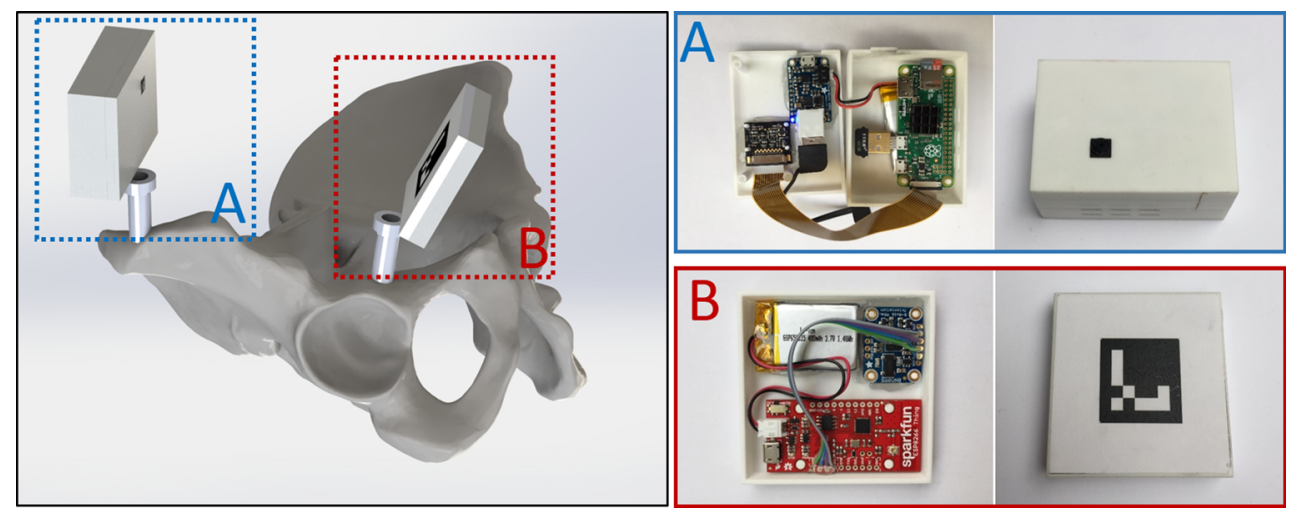

Fig. 1 The setup and components of our system. Left: The overall setup with the tracking unit (a) attached to the patient's pelvis facing toward the acetabular fragment where the augmented marker is attached (b).

camera module (Pi NoIR, Raspberry Pi Foundation, UK). It is directly placed on the patient's pelvis, facing toward the acetabular fragment area, and its only task is to stream live video to the host computer using a wireless connection (WiFi). All further video processing (marker detection, identification and pose estimation) is done on the host computer. 2) An augmented marker unit which consists of a printed planar Aruco marker [10] on the outer surface facing toward the tracking unit. On the inside, a WiFi system on a chip (SoC, ESP8266) with built-in full TCP/IP stack is combined with an IMU (BNO055, Robert Bosch GmbH, Germany) comprised of a triaxial gyroscope, triaxial accelerometer and a triaxial magnetometer with built-in sensor fusion. This IMU offers several sensor fusion modes including a magnetometer-less fusion mode that only makes use of the built-in accelerometers and gyroscopes. We chose this sensor fusion mode, to avoid any issue with magnetic field distortions which is crucial to use the system in an operating room (OR). 3) The host computer receives the video stream from the tracking unit and the IMU pose estimates from the augmented marker to compute a final pose estimate. The basic setup of the system is shown in Fig. 1. A schematic overview of the different components involved in the proposed system is shown in Fig. 2.

\section{Marker tracking}

The tracking unit's camera module must be first calibrated once. We do this using OpenCV's camera calibration framework. After the tracking unit connects to the host computer using a wireless connection, we start the marker tracking in a separate thread. The tracking unit provides gray scale images with a resolution of 540 by 960 pixels. The marker detection in each frame is implemented in $\mathrm{C} / \mathrm{C}++$ using OpenCV. We first apply thresholding and detect contours in the image.
Middle: Inside look at the tracking unit and the augmented marker. Right: Outside look at the tracking unit and the augmented marker

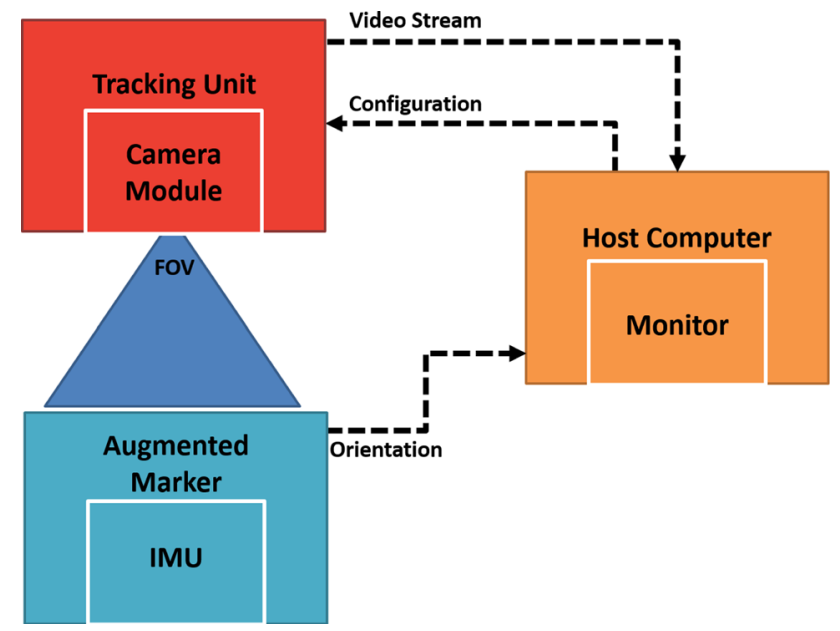

Fig. 2 A schematic illustration of how our system works. The tracking unit (red) sends a live video stream of the augmented marker to the host computer. The augmented marker (light blue) sends the orientation output from the IMU to the host computer (orange). The host computer detects the Aruco marker in the video stream and estimates its orientation using a robust pose estimation algorithm [48]. Sensor fusion is performed to fuse the pose estimate from the IMU with the pose estimate from the Aruco marker tracking

Contours are filtered by approximating each contour by a polygon and rejecting those which cannot be represented by four individual corner points (rectangles). The final marker candidates are rectified and filtered based on whether or not they represent the Aruco marker ID we are looking for $[10,43]$. The detection pipeline is illustrated in Fig. 3. The pose is computed using a robust pose estimation algorithm proposed by Schweighofer et al. [48]. Before we can apply any kind of sensor fusion, we must first compute the calibration matrix relating the Aruco marker's local coordinate system to the local coordinate system of the IMU which is running in parallel. This is a typical hand-eye calibration 


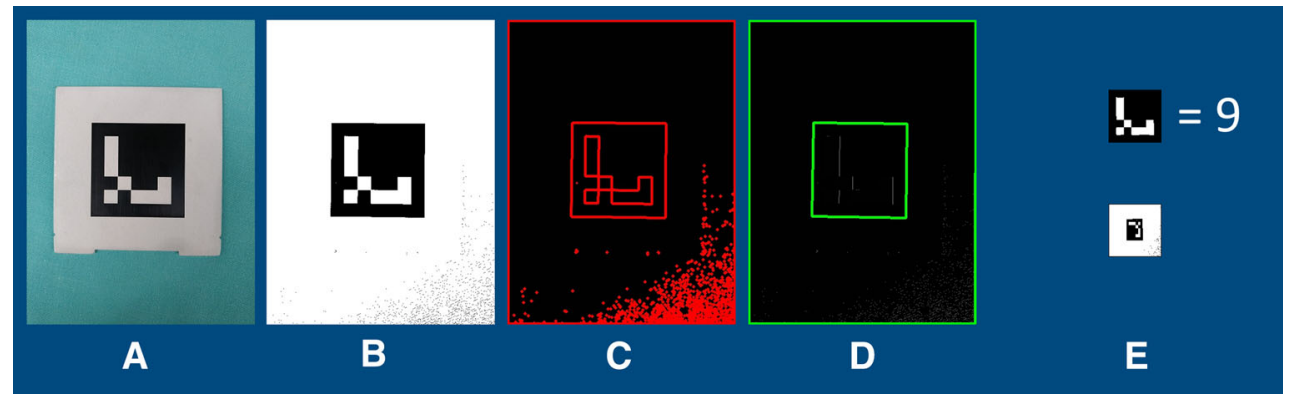

Fig. 3 Aruco marker detection pipeline. a Input image b. Thresholding to create a binary image. c All detected contours (red). d Marker candidates that can be represented as a polygon with exactly four cor- ner points (green). e Two rectified marker candidates. The top marker candidate can be successfully decoded, whereas the bottom candidate cannot be decoded and is therefore not a valid Aruco marker

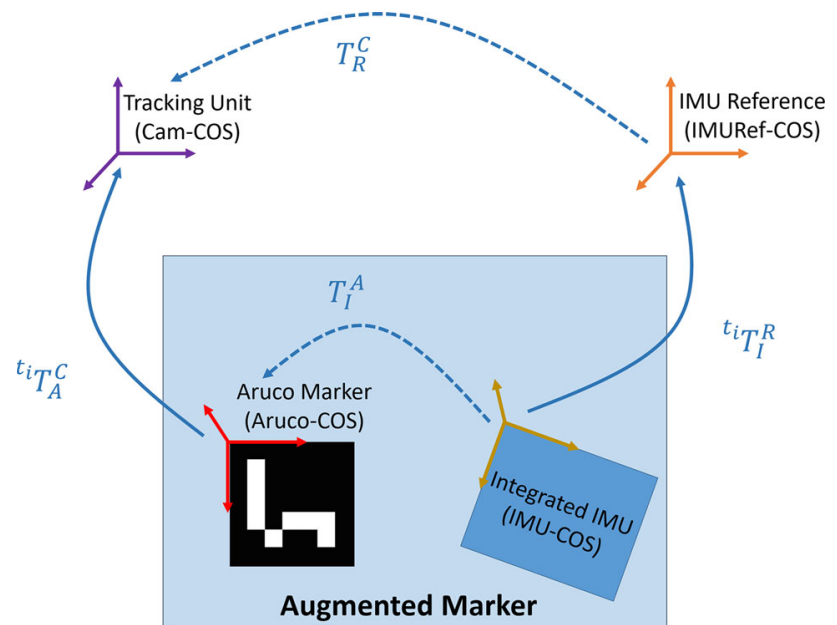

Fig. 4 All involved coordinate systems (COS). The calibration procedure is necessary to estimate the fixed transformation $T_{I}^{A}$ between the IMU's local coordinate system and the Aruco marker's local coordinate system. This procedure can be done once as the relation between the marker and the IMU stays fixed

problem [24]. The calibration procedure is shown in detail in the following section.

\section{Calibration}

The IMU is rigidly attached to the back of the planar marker. The involved coordinate systems are shown in Fig. 4. Our approach is based on a set of measurements of the planar marker tracking and the IMU. At every time point $t_{i}$, we receive the transformation from the planar marker's local coordinate system to the tracking unit's camera coordinate system $\left(T_{A}^{C}\right)$ as well as the transformation from the IMU coordinate system to its measurement reference space $\left(T_{I}^{R}\right)$. The goal of this calibration procedure is to estimate the transformation $T_{I}^{A}$ from the IMU local coordinate system to the Aruco marker's local coordinate system. At any two time points $t_{i}$ and $t_{j}$, we have the following relationships:

$T_{I}^{A}={ }^{t_{i}} T_{C}^{A} \cdot T_{R}^{C} \cdot{ }^{t_{i}} T_{I}^{R}$

and

$T_{I}^{A}={ }^{t_{j}} T_{C}^{A} \cdot T_{R}^{C} \cdot{ }^{t_{j}} T_{I}^{R}$

We have now two unknown transformations $T_{I}^{A}$ and $T_{R}^{C} . T_{R}^{C}$ can be eliminated and we obtain:

$T_{I}^{A}={ }^{t_{j}} T_{C}^{A} \cdot\left({ }^{t_{i}} T_{C}^{A}\right)^{-1} \cdot T_{I}^{A} \cdot\left({ }^{t_{i}} T_{I}^{R}\right)^{-1} \cdot{ }^{t_{j}} T_{I}^{R}$

Let's define

${ }^{t_{i j}} \Delta T_{C}^{A}={ }^{t_{j}} T_{C}^{A} \cdot\left({ }^{t_{i}} T_{C}^{A}\right)^{-1}$

and

${ }^{t_{i j}} \Delta T_{R}^{I}=\left({ }^{t_{j}} T_{I}^{R}\right)^{-1} \cdot{ }^{t_{i}} T_{I}^{R}$

Then we end up with:

$\left({ }^{t_{i j}} \Delta T_{C}^{A}\right)^{-1} \cdot T_{I}^{A}=T_{I}^{A} \cdot{ }^{t_{i j}} \Delta T_{R}^{I}$

We can now, without causing any confusion, arbitrarily select a time $t_{i}=t_{0}$. The incremental transformations defined in Eqs. 4 and 5 are measured with respect to this time point such that we can drop the index $i$. Only considering the rotational part of the transformation, we can reformulate as:

$\Delta R_{A}^{j} \cdot R_{I}^{A}=R_{I}^{A} \cdot \Delta R_{I}^{j}$

We end up with the classical hand-eye calibration equation of the form $A X=X B[24,41]$. Following [41], we will use Lie 
group theory to convert the problem to a least-squares problem where a closed-form solution is available. For a given rotation matrix $R$, we have:

$\theta=\arccos \left(\frac{\operatorname{trace}(R)-1}{2}\right)$

$\log (R)= \begin{cases}0, & \text { if } \theta=0 \\ \operatorname{skew}(r), & \text { if } \theta \neq 0 \text { and } \theta \in(-\pi, \pi)\end{cases}$

where $r=\theta a_{R}=\left[r_{x} r_{y} r_{z}\right]^{T}$ is the axis-angle representation of the rotation matrix $R$ and:

$\operatorname{skew}(r)=\left[\begin{array}{lll}0 & -r_{z} & r_{y} \\ r_{z} & 0 & -r_{x} \\ -r_{y} & r_{x} & 0\end{array}\right]$.

Applying this equation to $\Delta R_{I}^{j}, \Delta R_{A}^{j}$, we have:

$\log \left(\Delta R_{A}^{j}\right)=\operatorname{skew}\left(b_{j}\right)$

and

$\log \left(\Delta R_{I}^{j}\right)=\operatorname{skew}\left(a_{j}\right)$

According to Lie theory, we have:

$b_{j}=R_{I}^{A} a_{j}$

To handle noisy measurements, we can formulate our original problem as the following least-squares minimization problem:

$\min _{R_{I}^{A}} \sum_{j=1}^{n}\left|R_{I}^{A} a_{j}-b_{j}\right|^{2}$

where a closed-form solution can be calculated efficiently as

$R_{I}^{A}=U V^{-\frac{1}{2}} U^{-1} M^{T}$

With $M=\sum_{j=1}^{n} b_{j} a_{j}^{T}$, and the eigen-decomposition of $M^{T} M=U V U^{-1}$.

\section{Patient registration}

The goal of patient registration is to relate the virtual 3D model of the pelvis displayed on the host computer with the coordinate system of the patient. Since we cannot digitize landmarks the way it is usually performed with optical tracking-based navigation systems (tracked pointer), we record the orientation of the anterior pelvic plane and use its orientation to align the computer model accordingly. In this work, we will use a previously developed registration device
[42] that could be sterilized for future clinical application. The device's three equal length pillars are placed on the three landmarks (left and right anterior superior iliac spine (ASIS) and one of the two pubic tubercles) that define the APP. We can now align the augmented marker with the device's top plate which is now parallel to the APP. We use the integrated IMU to record the orientation of the APP. This way we do not have to assure that the Aruco marker is within the fieldof-view (FOV) of the tracking unit's camera, significantly improving convenience compared to our previous work [43]. The augmented marker is attached to the top plate so that its local $\mathrm{x}$-axis is aligned with the connection between the left and right ASIS. This way, the Aruco marker's local z-axis is representing the APP normal (see Fig. 5). We measure the orientation of the APP in the IMU reference coordinate system, and once the augmented marker is attached to the acetabular fragment (right before the reorientation starts), we have the required information available to transform the recorded APP orientation from the IMU reference coordinate system to the Aruco's local coordinate system using the previously computed fixed calibration matrix $T_{I}^{A}$.

\section{Sensor fusion}

So far, the host computer first detects the Aruco marker in each received video frame. Simultaneously, the IMU sends its orientation output to the host computer which we transformed to the planar marker's local coordinate system using the calibration matrix. As a reminder, the IMU has built-in sensor fusion which produces an orientation update at a high internal update rate $(100 \mathrm{~Hz})$. In the first prototype of the system, we fully trusted the orientation output from marker tracking as long as the marker was visible and only used the IMU during patient registration or in times when the marker was not visible in the video frame. This first prototype was validated using a cadaver study (more details in "Experiments" section). This binary decision scheme has the drawback that we do not exploit the high update rate from the IMU but simply wait for the update from the marker tracking (around 8$12 \mathrm{~Hz}$ ). It is therefore desirable to fuse the pose estimate from the IMU with the data from the marker tracking. We propose a sensor fusion approach which uses the IMU's pose estimate as a prediction for the location of the marker in the next video frame. We start off with marker detection as described in "Marker Tracking" section using simple thresholding with a fixed threshold value. If the marker was not detected, we repeat simple thresholding until the marker is detected. If the marker is detected (Aruco marker detected and identified ID is correct), we feed this information to a Kalman filter $[22,44]$ as the initial orientation estimate. Next, we use the orientation output from the IMU to predict the marker location in the next video frame, effectively reducing the search area. This allows us to use adaptive thresholding on this smaller search 


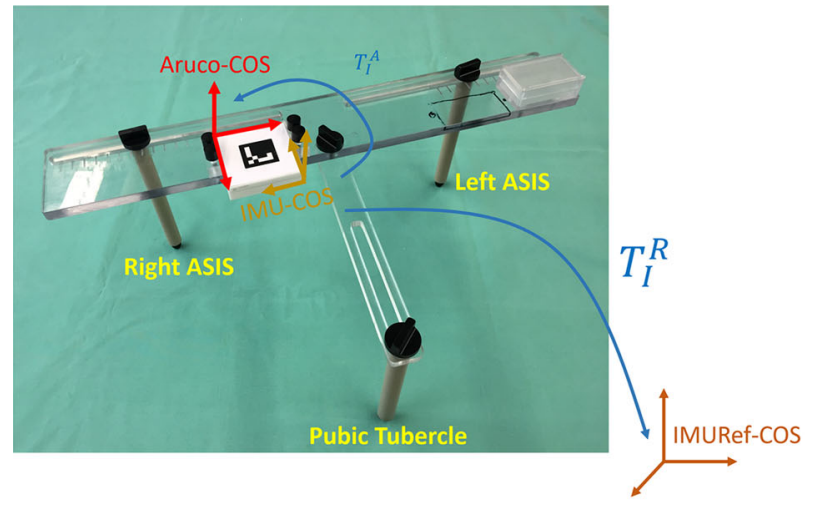

APP Registration

Fig. 5 Overview of the patient registration and the involved transformations. Left: The previously developed registration device is placed on the patient. The three pillars are placed on the right and left anterior superior iliac spine (ASIS) and on one of the two pubic tubercles. This aligns the top plate with the APP. The attached augmented marker is placed on the top plate in a way that its $\mathrm{x}$-axis is parallel to the connection between the right and left ASIS. The Aruco marker's local z-axis is

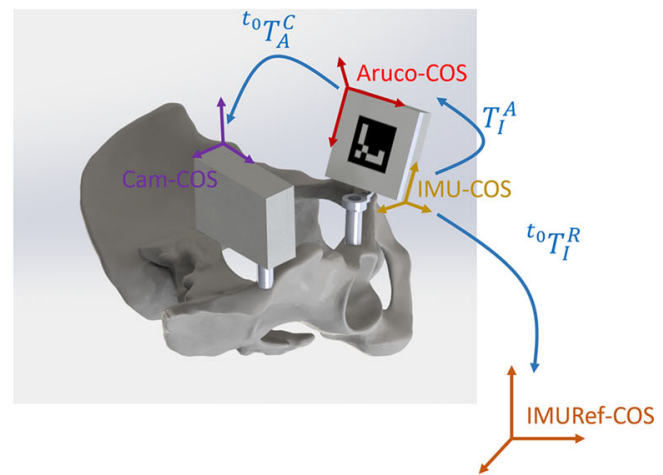

Before fragment reorientation now representing the APP normal. $T_{I}^{A}$ is the calibration transformation, and $T_{I}^{R}$ is measured by the IMU integrated into the augmented marker. Right: Right before the acetabular fragment is detached, we will have all necessary information available to bring the measured APP orientation into the planar marker's local coordinate system where we later perform all computations during acetabular reorientation

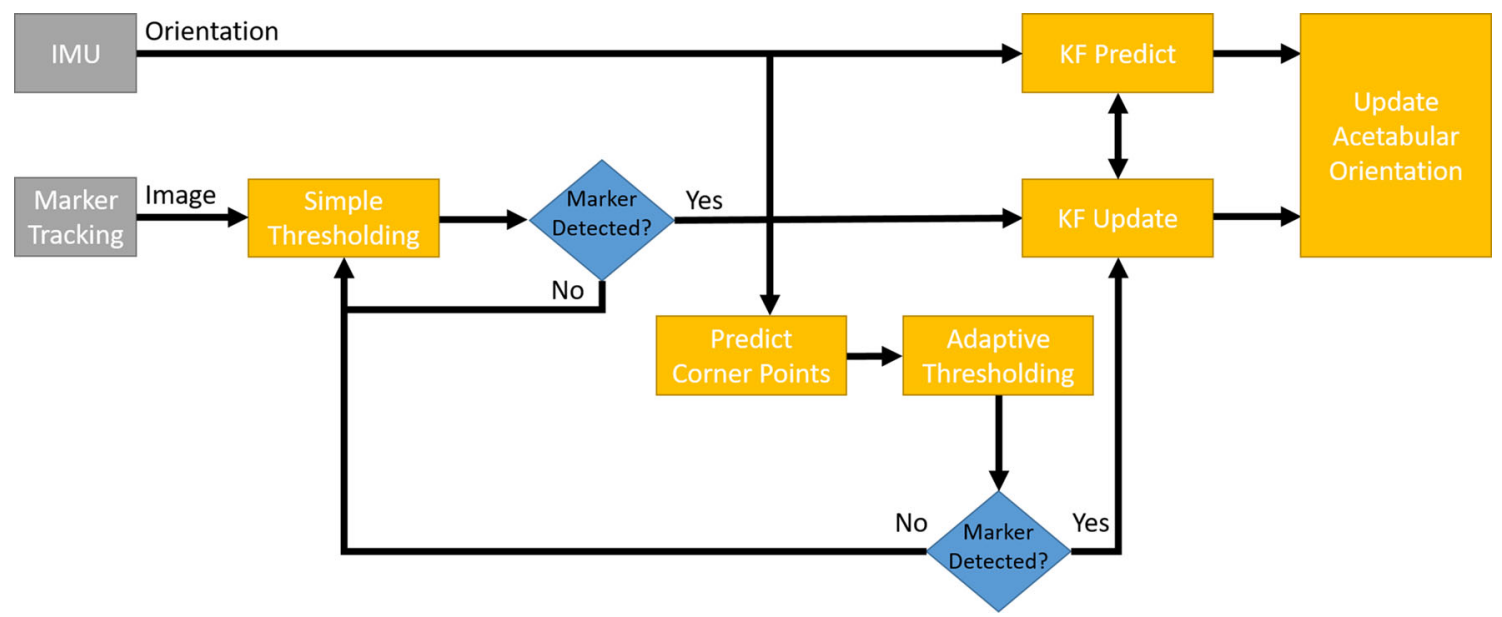

Fig. 6 Overview of the proposed sensor fusion algorithm. The Kalman filter is initialized using the first pose estimate from the Aruco marker tracking using simple thresholding. Next, we use the output from the IMU directly as the prediction for the Kalman filter and also to predict the next location of the Aruco marker in the input image. This prediction allows us to restrict the search area and to apply adaptive thresholding which is computationally less efficient than simple thresholding. If the marker is detected, we update the Kalman filter and continue with this strategy. If, however, the marker was not detected within the smaller search area, then we simply fall back to the simple thresholding over the whole image area without reducing performance. If the marker is detected, we compute its pose and feed the output to the Kalman filter. If the marker was not detected (no Aruco marker detected and/or ID not correct) in that smaller search area using adaptive thresholding (e.g., fast movement), we then simply fall back to the simple thresholding over the whole image. In this case, the Kalman filter never leaves the prediction state and we rely on the input from the IMU. As soon as the Aruco marker is detected in the whole image, we continue with the update state of the Kalman filter. The whole sensor fusion pipeline is outlined in Fig. 6. The Kalman filter uses the output from the IMU directly as the prediction. The output from the marker tracking serves as the measurement update. During prediction, instead of using the previous state, we simply replace the state vector with the output from the IMU. This reduces the state transition matrix to an identity matrix. The prediction equations of the Kalman filter are shown in Eqs. 16 and 17. 
$q_{k}=A_{k} q_{k}^{I M U}$

$P_{k \mid k-1}=A_{k} P_{k-1 \mid k-1} A_{k}^{T}+Q_{k}$

$A_{k}$ is the state transition matrix and $P_{k}$ is the state covariance matrix. $Q_{k}$ is the process noise covariance matrix. The prediction step is repeated as long as there is no update from the marker tracking. As soon as the marker detection is successful, the following update equations are applied:

$y_{k}=q_{k}^{\text {Marker }}-H_{k} q_{k}$

$S_{k}=H_{k} P_{k \mid k-1} H_{k}^{T}+R_{k}$

$K_{k}=P_{k \mid k-1} H_{k}^{T} S_{k}^{-1}$

$q_{k}=q_{k}+K_{k} y_{k}$

$P_{k \mid k}=\left(I-K_{k} H_{k}\right) P_{k \mid k-1}$

where $y_{k}$ is the innovation, $S_{k}$ the innovation covariance matrix, $H_{k}$ is the observation matrix (set to identity) and $K_{k}$ is the Kalman gain.

\section{Experiments}

We performed two plastic bone studies to evaluate each tracking technology individually. One with only Aruco marker tracking (no IMU, except patient registration) and the other with only IMU tracking. In addition, we performed a cadaver study with a binary decision scheme for sensor fusion and a plastic bone study using the proposed sensor fusion approach.

For the cadaver study, we used simple thresholding over the whole image at all times to detect the marker. Every time the marker was detected, we fully trust the pose estimate from marker tracking, ignoring the input from the IMU. Only during times when the marker was not detected, we fully relied on the pose estimation from the IMU. No Kalman filter was applied, and the pose estimates were used directly.

The following subsections describe all the experiments in more detail.

\section{Plastic bone study—single tracking technology}

Two validation plastic bone studies including 3 pelvises (6 hip joints) were performed sequentially to validate each tracking technology individually. Simultaneously, we ran a previously developed and validated optical tracking-based navigation system (Polaris, NDI Canada) serving as ground truth measurements [31]. Inclination and anteversion values of the two navigation systems for PAO were compared during acetabular reorientation. We oriented the fragment to 20 random positions and simultaneously recorded inclination and anteversion values from both systems. For the marker-only tracking, we still used the IMU to perform patient registration. Only during acetabular reorientation, we fully relied on marker tracking.

\section{Cadaver study}

A validation study including 3 pelvises (6 hip joints) undergoing navigated PAO was performed. All six PAOs were performed by a hip surgeon with experience in hip preservation surgery (MT), and acetabular reorientations were performed by a first-year resident in orthopedic surgery (TL). We used our proposed augmented marker trackingbased navigation system with a binary decision scheme to measure the acetabular orientation. The same ground truth system as described above was used for validation. Again, we reoriented the fragment to 20 random positions and recorded inclination and anteversion values from both systems simultaneously. All necessary steps (patient registration and fragment reorientation) were repeated three times (A, B and C), resulting in a total of 360 different reorientation positions. The setup during the cadaver study was organized to simulate a clinical application (see Fig. 7).

\section{Plastic bone study—sensor fusion}

A validation plastic bone study including 3 pelvises (6 hip joints) was performed to validate the system using the proposed sensor fusion method. The same optical tracking-based system as used during the other plastic bone studies and the cadaver study served as ground truth. Again, the acetabular fragment was reoriented to 20 random positions and we recorded inclination and anteversion from both systems simultaneously resulting in 120 different reorientation positions.

\section{Statistical evaluation}

We computed mean absolute difference and correlation between the proposed system and the optical tracking-based ground truth system [31] for anteversion and inclination separately. A box plot showing the median difference and the 5-th and 95-th percentile was also computed for anteversion and inclination separately. Bland-Altman plots were computed for the results of the cadaver study to make sure that no systematic error was present in the study.

\section{Results}

\section{Plastic bone study—single tracking technology results}

For the marker-tracking plastic bone study, we achieve a mean absolute difference for inclination and anteversion of $1.71( \pm 1.49)$ and $1.09( \pm 1.17)^{\circ}$, respectively. For the IMU-based tracking, we achieve a mean absolute difference 
Fig. 7 Experimental setup: Left: Close-up view of the tracking unit (a) and the augmented marker (b) attached to the pelvis and fragment, respectively. For validation purposes, the augmented marker and the tracking unit were attached to the DRBs used by the optical tracking-based navigation system. Right: The tracking unit (a) is attached to the pelvis and the augmented marker (b) is attached to the acetabular fragment. The optical tracking stereo camera (c) and the host computer (d) are shown in the background

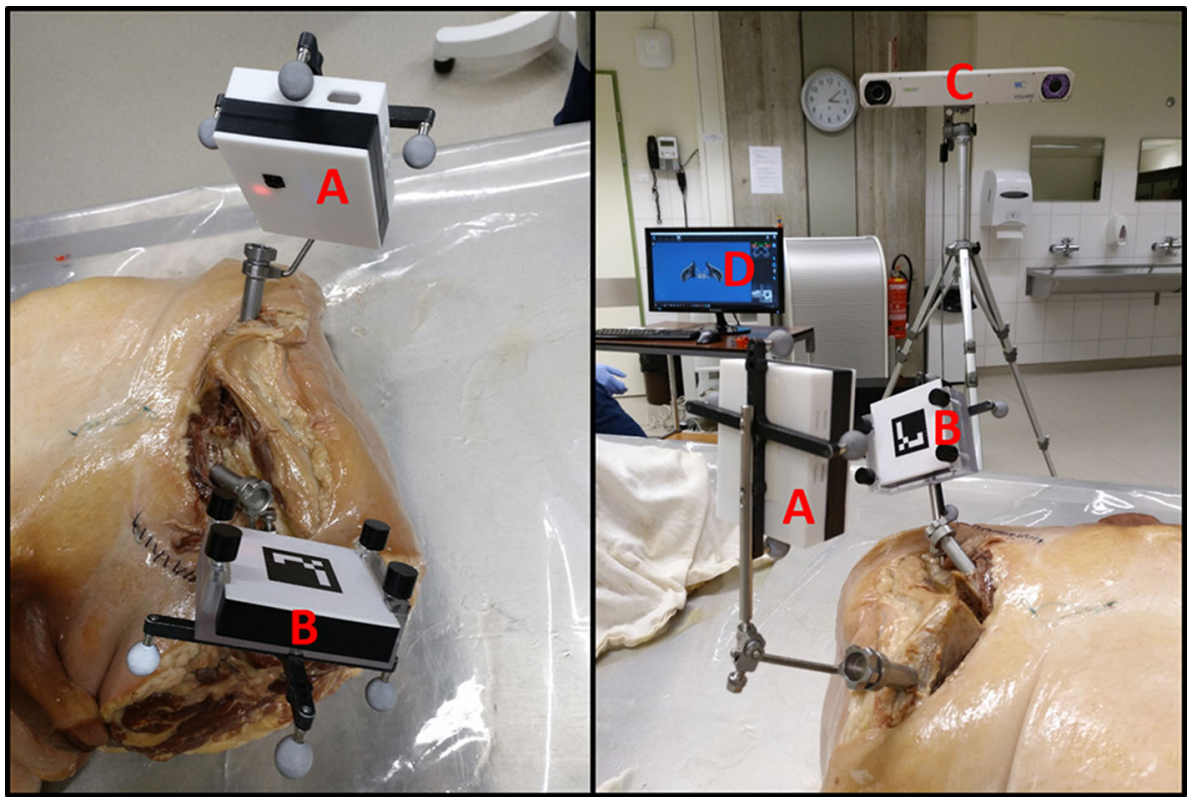

for inclination and anteversion of $3.23( \pm 3.79)$ and 2.76 $( \pm 2.27)^{\circ}$, respectively. We performed a Wilcoxon rank sum test for both tracking technologies, comparing the inclination and anteversion values measured by each individual tracking technology to the values measured by the optical tracking system. The resulting $p$-values comparing inclination and anteversion values are $(0.8140 / 0.7246)$ for marker-based tracking and (0.9311/0.5562) for IMU-based tracking. In all cases, we cannot reject the null-hypothesis at the 5\% significance level. The correlation is very strong for both tracking technologies. We achieve a correlation for inclination and anteversion of $(0.99 / 0.99)$ for marker-based tracking and $(0.97 / 0.93)$ for IMU-based tracking. In terms of update rate, we achieve an update rate of around $8-12 \mathrm{~Hz}$ for marker tracking, depending on the number of detected marker candidates and the pose of the marker relative to the camera sensor plane [48]. The IMU has an internal update rate of $100 \mathrm{~Hz}$, and measuring the update on the host computer during acetabular reorientation, we achieve an update rate of around $35 \mathrm{~Hz}$.

\section{Cadaver study results}

Mean absolute difference for inclination and anteversion $(N$ $=360)$ was $1.34( \pm 1.50)$ and $1.21( \pm 1.07)^{\circ}$, respectively. The measurements from our system show a very strong correlation to the ground truth optical tracking-based navigation system for both inclination and anteversion (0.98/0.97). Figure 8 shows a box plot with the median error and the 5th and 95-th percentiles for each individual case. Figure 9 shows the Bland-Altman plots for anteversion and inclination.

\section{Plastic bone study—sensor fusion results}

Using the proposed sensor fusion method, we achieved a very strong correlation to the ground truth optical tracking-based navigation system $(0.99 / 0.96)$. Mean absolute difference for inclination and anteversion $(N=120)$ was $1.63( \pm 1.48)$ and $1.55( \pm 1.49)^{\circ}$, respectively. Figure 10 shows the box plot for the plastic bone study results using sensor fusion, for each individual case for the 6 hip joints.

\section{Discussion}

We successfully demonstrated the feasibility and clinical applicability of our PAO navigation system to measure inclination and anteversion during acetabular reorientation. We compared our system to a gold-standard optical trackingbased navigation system with a plastic bone study and a cadaver study.

Strong correlation and small mean absolute differences for both anteversion and inclination were found in the cadaver study and the plastic bone study (with the proposed sensor fusion). Advantages of this hybrid system include minimal space requirements for the setup in the OR and no LOS impediment.

Using a navigation system offers several benefits as reported by others $[16,27,33]$, especially for inexperienced surgeons. Intra-operative manual measurements of acetabular fragment positioning can be error prone. Murphy et al. [33] compared intra-operative manual measurements with postoperative $\mathrm{CT}$ measurements, and they reported relative large errors in measuring the adduction angle of $10.5^{\circ}$. The measurements of their proposed biomechanical guidance 

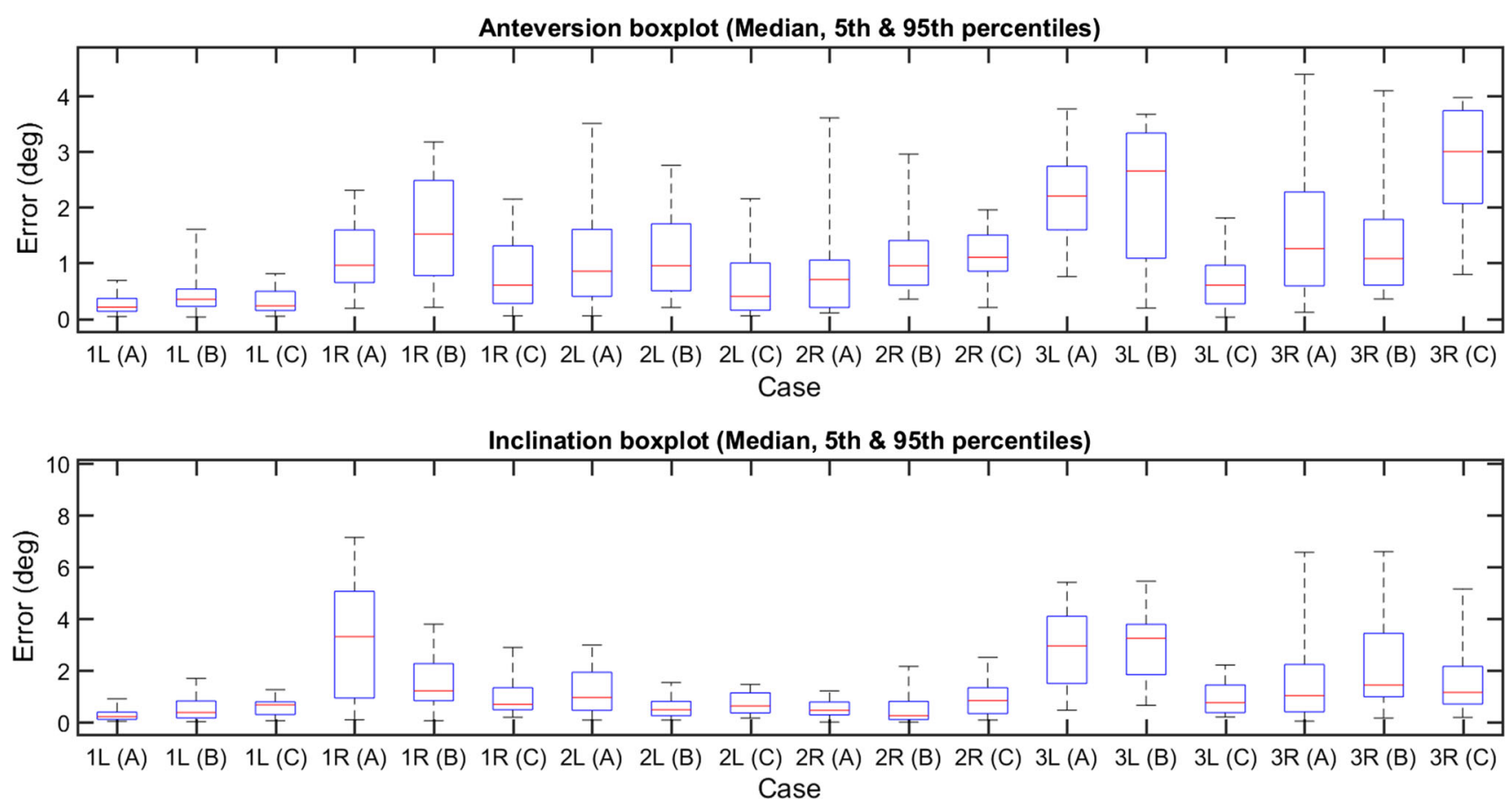

Fig. 8 Error box plots showing the median error and the 5-th and 95-th percentiles for each individual case of the cadaver study. Letters L/R represent joint side. Letters A, B and C represent the first, second and third experiment for a specific hip joint. Top: Anteversion Bottom: Inclination

system showed a strong agreement to the CT measurements (mean of $3.7^{\circ}$ ) [33]. These errors are slightly larger than traditional optical tracking-based navigation systems. Liu et al. [30] reported errors of decomposed motion components of less than $0.6-0.9^{\circ}$ between the pre-operative plan and the intra-operative measured results. However, they did not compare to postoperative CT measurements.

We first performed two plastic bone studies to evaluate the performance of each individual tracking technology. The results show a difference in performance between marker tracking and IMU-based tracking. The IMU has built-in sensor fusion which uses the integrated triaxial gyroscope and triaxial accelerometer to compute a final pose estimate. We decided not to use the sensor fusion mode with the magnetometer, as the magnetic field is not constant in an operating room and can disturb the final pose estimate significantly $[40,42]$. Using the gyroscope to estimate the angle around the gravity vector is prone to drift as we cannot correct for it using the accelerometers. It is therefore not surprising that the IMU-based tracking performed worse than the markerbased tracking. The IMU-only tracking also is based on the assumption that the pelvis does not move with respect to the IMUs reference coordinate system. We achieve a mean absolute difference of less than $4^{\circ}$ but with a relatively high standard deviation. These results are not surprising compared to other work using IMUs for navigation. Behrens et al. [2] report errors in the range of $0.4-3.4^{\circ}$ for endoscope tracking. Walti et al. [53] also report errors in the range of $2-4^{\circ}$ for pedicle screw placements using an IMU as guidance.

The first two plastic bone studies show that it is advantageous to combine both tracking technologies (marker tracking and IMU), as marker-based tracking has a limited working volume based on the FOV of the camera and a lower update rate but a higher accuracy compared to IMU tracking.

We performed a cadaver study with a binary decision scheme, fully trusting the marker tracking unless the marker was not detected. The overall results are very promising. This approach works well in cases where the marker rarely goes outside the camera's FOV. This is most often the case, especially during the cadaver study when range of motion is limited by soft-tissue constraints.

Katanacho et al. [23] used QR codes and a high-resolution HD camera (1280 x 1024px) attached to a lamp to track surgical instruments. Attaching the camera to the operating room lamp has the advantage of an easier setup and the possibility to send the video frames through a wired connection. On the other hand, the lamp cannot be moved during surgery and has generally a larger distance to the tracked tools, requiring larger markers. Compared to their work, we use a lowerresolution camera directly attached to the patient's pelvis and an Aruco marker instead of QR codes. The advantage of using QR codes is the possibility to store additional information such as marker size and marker-to-tip transformation but they are generally harder to detect and identify. Encoding additional information in the marker is not necessary 

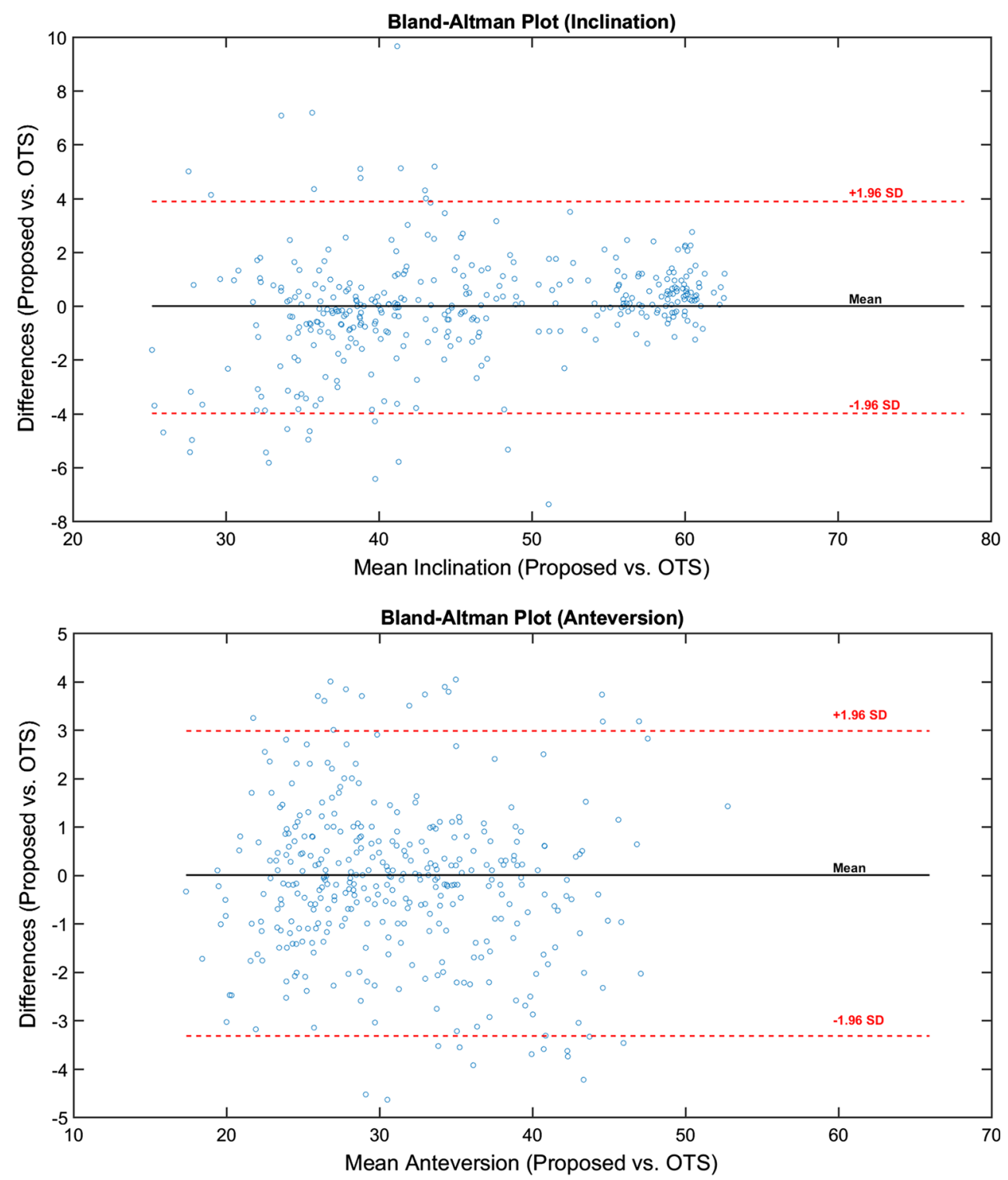

Fig. 9 The Bland-Altman plots for inclination (top) and anteversion (bottom) of the cadaver study. A high level of agreement can be observed between the measurements from the proposed system compared to the measurements from the ground truth optical tracking-based navigation system

in our case, as we just use one marker and only rely on rotational information during tracking and no marker-to-tip transformation is needed. Katanacho et al. report a lower accuracy for rotation measurements of around $2-5^{\circ}$ depending on marker size and distance. One reason for the lower accuracy is the error-prone detection process for computing point correspondences [23]. Camera-to-marker distance is in fact important for accurate planar marker tracking. Errors in computing the pose have several sources including errors in marker detection due to noise, changing lighting conditions, camera calibration errors, occlusions and errors from the pose estimation algorithm itself [9]. In our case, the distance from the camera to the marker is limited by the anatomical con- straints and it is possible to use different marker sizes should the distance be large.

The third plastic bone study we performed included the proposed sensor fusion approach which uses the IMU output as a prediction for the location of the marker in the image and used the marker tracking output to correct the Kalman filter prediction. This allows us to reduce the search area in the image giving us the possibility to apply adaptive thresholding instead of simple thresholding. This is more robust in difficult lighting conditions as adaptive thresholding generally gives better results than simple thresholding (especially around the marker corners). 

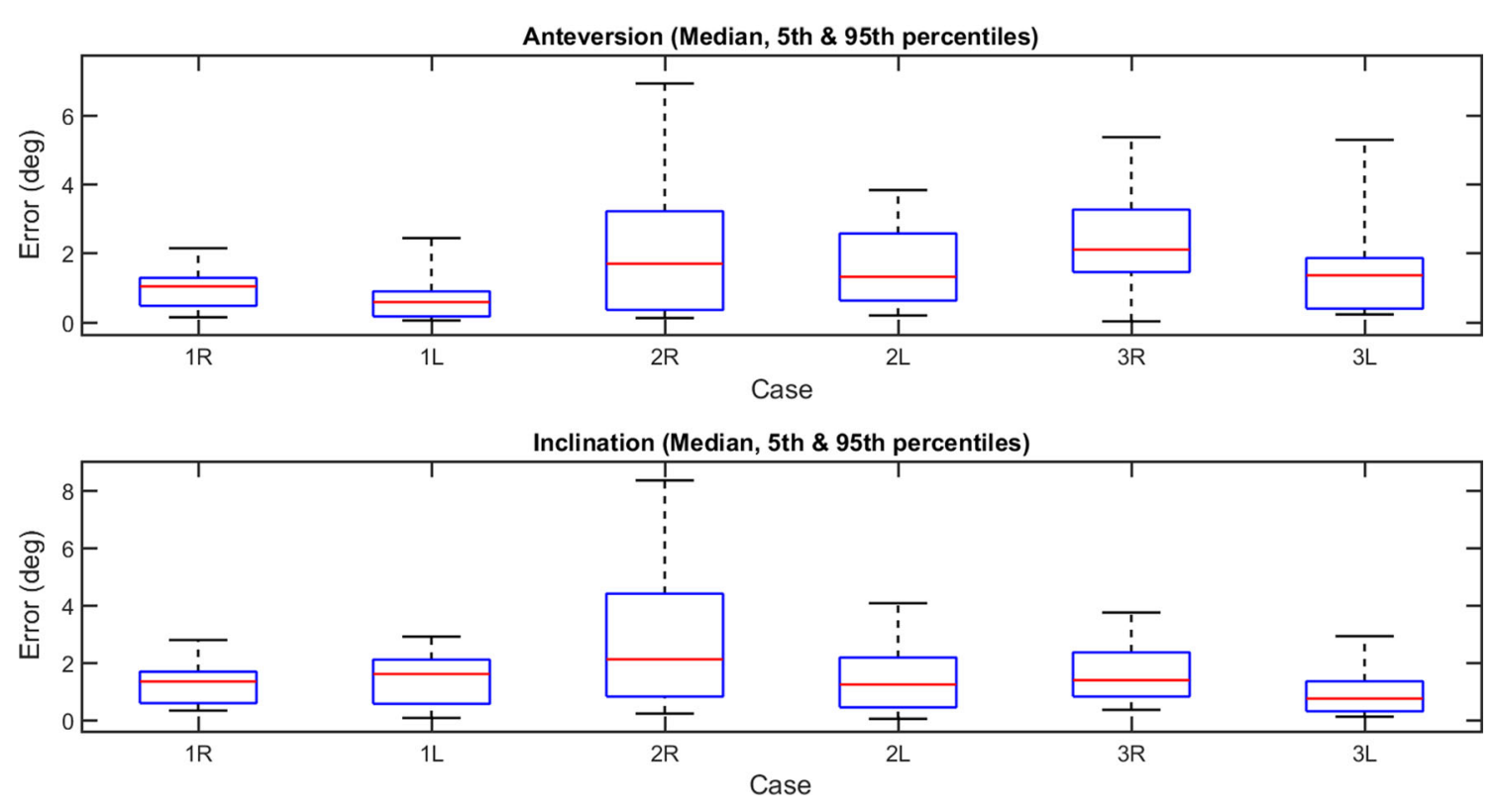

Fig. 10 Box plots showing the median error and the 5-th and 95-th percentiles for each individual case for the 6 hip joints of the plastic bone study compared to the optical tracking-based navigation system. Letters L/R represent joint side. Top: Anteversion Bottom: Inclination

Fig. 11 The manufactured augmented marker that can be directly attached to the T-handle used during PAO surgery. The marker box is designed in a way that it can easily be attached to the top part of the T-handle. In this way, the reorientation can be directly performed without the need to drill an additional screw hole into the acetabular fragment. The box is made from polyether ether ketone (PEEK) and the marker is laser printed on to a 2-mm aluminum sheet that can be inserted on the outside
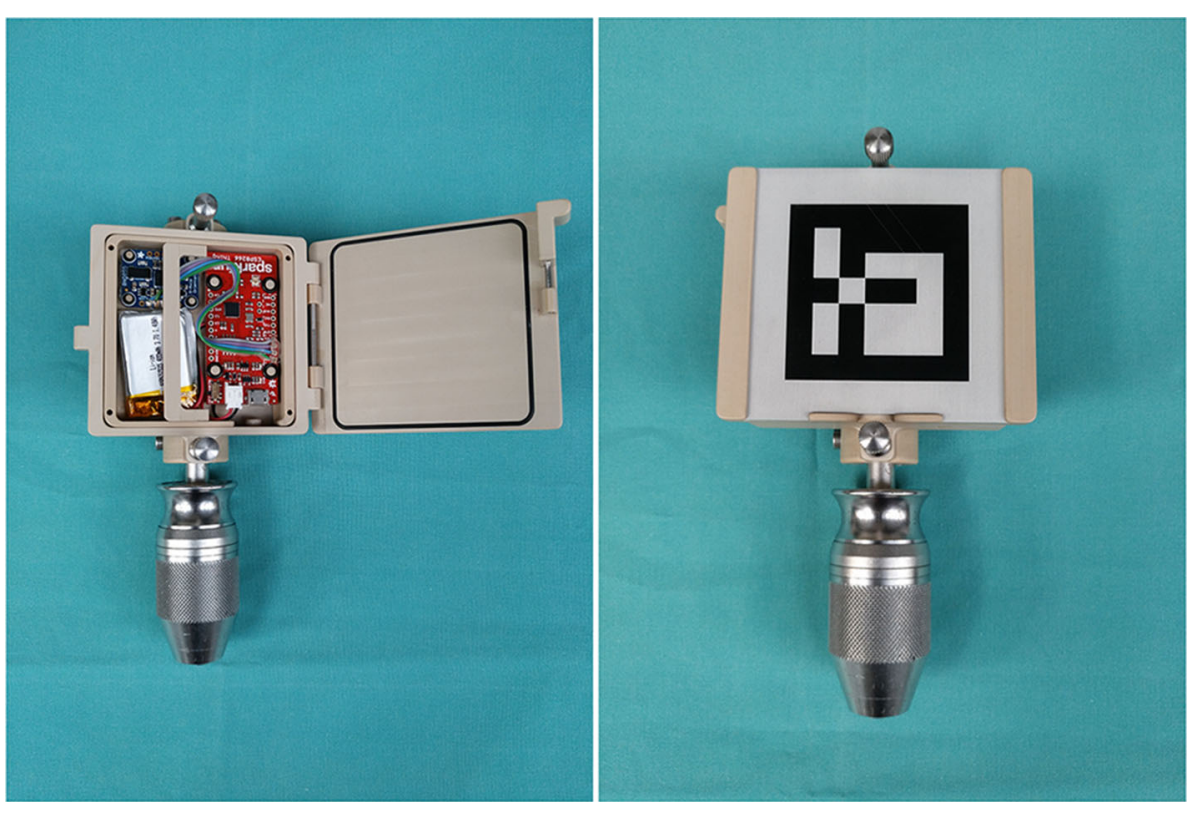

Our work has limitations. During patient registration, we solely rely on the IMU measurements. This allows a convenient patient registration procedure as we do not have to make sure that the planar marker is visible in the tracking unit's FOV. We use the sensor fusion mode provided by the IMU that only makes use of the gyroscopes and accelerometers. This has the advantage that the measurements are not susceptible to magnetic field changes, but has the disadvantage that drift errors from the integration of the gyroscope signal can affect the accuracy significantly over time. Cao et al. [5] recently presented a similar system to measure the pose of the APP for total hip arthroplasty. They validated their system using plastic bones and reported errors of less than $1.2^{\circ}$. During the cadaver study, patient registration only took around 30-60s, reducing the effects of drift. During reorientation, drift is negligible as the update from the planar marker tracking is able to correct for any drift errors in the prediction step of the Kalman filter.

The cadaver study shows a smaller error than the plastic bone studies (mean difference of 1.34 vs. 1.63 for inclination and 1.21 vs. 1.55 for anteversion). One possible explanation is the limited range of motion when reorienting the acetabular 
fragment. Soft tissue around the hip joint prevents excessive range of motion and prevents fast and shaky movements that can be frequently present in plastic bone studies as the fragment is completely separated from the rest of the pelvis without soft-tissue constraints.

We compared our system with the current gold standard (optical tracking). The same errors which are present in optical tracking-based navigation systems (soft tissue between digitization pointer and bony landmarks during registration) are therefore also present in this study. A limitation is that no postoperative $\mathrm{CT}$ scan could be performed.

The proposed system is directly attached to the patient's pelvis and acetabular fragment. Electronic devices that are close to the patient cause inconvenience (blood splatter, space and sterility). The system that was used is a prototype that uses readily available parts. Not all of these parts are suitable to be used in a future clinical study. The tracking unit uses a Raspberry Pi Zero W which runs a full operating system in the background which is not efficient given its task of simply streaming live video to the host computer. This could be replaced by using a custom field-programmable gate array (FPGA) board with a miniature camera.

We are progressing further in terms of sterilization and clinical application. The augmented marker, for example, can be directly attached to the T-handle that is regularly used during PAO surgery (see Fig. 11) [28]. For clinical applications, the electronics are placed in a smaller box which can be slid into the sterilized outer box. A similar inner/outer box setup can be imagined for the tracking unit. In terms of blood splatter, as the marker is printed onto a sterilizable material (e.g., aluminum sheet), it is simply possible to clean the blood splatters from its surface. However, since the acetabular fragment will already be cut when the system is used, we do believe that blood splatters should not be a major problem during acetabular reorientation.

The material for the outer boxes is polyether ether ketone (PEEK) which is a highly durable, robust and most importantly, sterilizable material. This material also allows the WiFi signal to penetrate it, making it easier since no external antenna must be designed.

\section{Conclusion}

We successfully demonstrated the feasibility of our augmented marker-based navigation system. Our proposed system is able to accurately estimate the acetabular orientation during PAO surgery without the line-of-sight impediment that state-of-the-art optical tracking-based navigation systems suffer from. Clinical application of this system could help the surgeon during PAO and improve patient outcome through proper acetabular reorientation.
Acknowledgements All procedures performed in studies involving human participants were in accordance with the ethical standards of the institutional and/or national research committee and with the 1964 Helsinki declaration and its later amendments or comparable ethical standards

Funding This study was funded by the Swiss National Science Foundation (Grant number 205321_163224).

\section{Compliance with ethical standards}

Conflict of interest The authors declare that they have no conflict of interest.

Informed consent Informed consent was obtained from all individuals included in the study.

\section{References}

1. Albers CE, Steppacher SD, Ganz R, Tannast M, Siebenrock KA (2013) Impingement adversely affects 10-year survivorship after periacetabular osteotomy for DDH hip. Clin Orthop Relat Res 471(5):1602-1614

2. Behrens A, Grimm J, Gross S, Aach T (2011) Inertial navigation system for bladder endoscopy. In: Proceedings of the annual international conference of the IEEE engineering in medicine and biology society, EMBS pp 5376-5379

3. Beller S, Eulenstein S, Lange T, Hünerbein M, Schlag PM (2009) Upgrade of an optical navigation system with a permanent electromagnetic position control: a first step towards "navigated control" for liver surgery. J Hepato Biliary Pancreat Surg 16(2):165-170

4. Bugbee WD, Kermanshahi AY, Munro MM, McCauley JC, Copp SN (2014) Accuracy of a hand-held surgical navigation system for tibial resection in total knee arthroplasty. Knee 21(6):1225-1228. https://doi.org/10.1016/j.knee.2014.09.006

5. Cao Z, Su S, Chen H, Tang H, Zhou Y, Wang Z (2016) Pose measurement of Anterior Pelvic Plane based on inertial measurement unit in total hip replacement surgeries. In: Proceedings of the annual international conference of the IEEE engineering in medicine and biology society, EMBS 2016-Octob, pp. 5801-5804

6. Claasen GC, Martin P, Picard F (2011) High-bandwidth lowlatency tracking using optical and inertial sensors. In: ICARA 2011-Proceedings of the 5th international conference on automation, robotics and applications, pp 366-371

7. Crockarell J, Trousdale RT, Cabanela ME, Berry DJ (1999) Early experience and results with the periacetabular osteotomy.pdf. Clin Orthop Relat Res 363:45-53

8. Desseaux A, Graf P, Dubrana F, Marino R, Clavé A (2016) Radiographic outcomes in the coronal plane with iASSIST versus optical navigation for total knee arthroplasty: a preliminary case-control study. Orthop Traumatolo Surg Res 102(3):363-368. https://doi. org/10.1016/j.otsr.2016.01.018

9. Freeman RM, Julier SJ, Steed AJ (2007) A method for predicting marker tracking error. In: 2007 6th IEEE and ACM international symposium on mixed and augmented reality, ISMAR, pp 157-160

10. Garrido-Jurado S, Muñoz-Salinas R, Madrid-Cuevas FJ, MarínJiménez MJ (2014) Automatic generation and detection of highly reliable fiducial markers under occlusion. Pattern Recognit 47(6):2280-2292

11. Gharaibeh MA, Solayar GN, Solayar GN, Harris IA, Chen DB, MacDessi SJ (2017) Accelerometer-based, portable navigation 
(kneealign) vs conventional instrumentation for total knee arthroplasty: a prospective randomized comparative trial. J Arthroplast 32(3):777-782. https://doi.org/10.1016/j.arth.2016.08.025

12. Goh GSH, Liow MHL, Lim WSR, Tay DKJ, Yeo SJ, Tan MH (2016) Accelerometer-based navigation is as accurate as optical computer navigation in restoring the joint line and mechanical axis after total knee arthroplasty. A prospective matched study. J Arthroplast 31(1):92-97. https://doi.org/10.1016/j.arth.2015.06.048

13. Goh GSH, Liow MHL, Tay DKJ, Lo NN, Yeo SJ, Tan MH (2017) Accelerometer-based and computer-assisted navigation in total knee arthroplasty: a reduction in mechanical axis outliers does not lead to improvement in functional outcomes or quality of life when compared to conventional total knee arthroplasty. J Arthroplast pp. 1-7. http://linkinghub.elsevier.com/retrieve/pii/ S0883540317307891

14. Haid M, Kamil M, Chobtrong T, Guenes E (2013) Machinevision-based and inertial-sensor-supported navigation system for the minimal invasive surgery. In: AMA conferences-SENSOR

15. Hipp J, Sugano N, Millis M, Murphy S (1999) Planning acetabular redirection osteotomies based on joint contact pressures. Clin Orthop Relat Res 364:134-143

16. Hsieh PH, Chang YH, Shih CH (2006) Image-guided periacetabular osteotomy: computer-assisted navigation compared with the conventional technique: a randomized study of 36 patients followed for 2 years. Acta Orthop 77(4):591-597

17. Huang EH, Copp SN, Bugbee WD (2015) Accuracy of a handheld accelerometer-based navigation system for femoral and tibial resection in total knee arthroplasty. J Arthroplast 30(11):19061910. https://doi.org/10.1016/j.arth.2015.05.055

18. Jaeger M, Westhoff B, Wild A, Krauspe R (2004) Computerassisted periacetabular triple osteotomy for treatment of dysplasia of the hip. Zeitschrift fur Orthopadie und Ihre Grenzgebiete 142(1):51-59

19. Jolles BM, Genoud P, Hoffmeyer P (2004) Computer-assisted cup placement techniques in total hip arthroplasty improve accuracy of placement. Clin Orthop Relat Res 426:174-9

20. Jost GF, Walti J, Mariani L, Cattin P (2016) A novel approach to navigated implantation of S-2 alar iliac screws using inertial measurement units. J Neurosurg Spine 24(3), 447-53. http://www. ncbi.nlm.nih.gov/pubmed/26565762

21. Jost GF, Walti J, Mariani L, Schaeren S, Cattin P (2017) Inertial measurement unit-assisted implantation of thoracic, lumbar, and sacral pedicle screws improves precision of a freehand technique. World Neurosurg 103:11-18. https://doi.org/10.1016/ j.wneu.2017.02.079

22. Kalman RE (1960) A new approach to linear filtering and prediction problems. J Basic Eng 82(1):35-45

23. Katanacho M, De la Cadena W, Engel S (2016) Surgical navigation with QR codes. Curr Dir Biomed Eng 2(1):355-358

24. Kim SJ, Jeong MH, Lee JJ, Lee JY, Kim KG, You BJ, Oh SR (2010) Robot head-eye calibration using the minimum variance method. In: 2010 IEEE International Conference on Robotics and Biomimetics, ROBIO 2010, pp. 1446-1451

25. Kruecker J, Viswanathan A, Borgert J, Glossop N, Yang Y, Wood BJ (2005) An electro-magnetically tracked laparoscopic ultrasound for multi-modality minimally invasive surgery. Int Congr Ser 1281:746-751

26. Langlotz F, Bächler R, Berlemann U, Nolte LP, Ganz R (1998) Computer assistance for pelvic osteotomies. Clin Orthop Relat Res 354(354):92-102

27. Langlotz F, Stucki M, Bächler R, Scheer C, Ganz R, Berlemann U, Nolte LP (1997) The first twelve cases of computer assisted periacetabular osteotomy. Comput Aided Surg 2(6):317-326

28. Lerch TD, Steppacher SD, Liechti EF, Siebenrock KA, Tannast M (2016) Periazetabuläre Osteotomie nach Ganz. Der Orthopäde 45(8):687-694
29. Lerch TD, Steppacher SD, Liechti EF, Tannast M, Siebenrock KA (2017) One-third of hips after periacetabular osteotomy survive 30 years with good clinical results, no progression of arthritis, or conversion to THA. Clin Orthop Relat Res 475(4):1154-1168

30. Liu L, Ecker T, Schumann S, Siebenrock K, Nolte P, Zheng G, Periacetabular I, The M (2013) Cadaveric validation of a novel planning and navigation system for peri-acetabular osteotomy ( PAO ). In: CURAC pp 26-29

31. Liu L, Ecker T, Schumann S, Siebenrock K, Nolte L, Zheng G (2014) Computer assisted planning and navigation of periacetabular osteotomy with range of motion optimization. Lecture Notes in Computer Science (including subseries Lecture Notes in Artificial Intelligence and Lecture Notes in Bioinformatics) 8674 LNCS(PART 2), pp 643-650

32. Mahfouz MR, Kuhn MJ, To G, Fathy AE (2009) Integration of UWB and wireless pressure mapping in surgical navigation. IEEE Trans Microw Theory Tech 57(10):2550-2564

33. Murphy R, Armiger R, Lepistö J, Armand M (2016) Clinical evaluation of a biomechanical guidance system for periacetabular osteotomy. J Orthop Surg Res (36). https://doi.org/10.1186/ s13018-016-0372-3

34. Murphy RJ, Armiger RS, Lepistö J, Mears SC, Taylor RH, Armand M (2015) Development of a biomechanical guidance system for periacetabular osteotomy. Int J Comput Assist Radiol Surg 10(4):497-508

35. Myers SR, Eijer H, Ganz R (1999) Anterior femoroacetabular impingement after periacetabular osteotomy. Clin Orthop Relat Res 363:93-9

36. Nam D, Jerabek SA, Haughom B, Cross MB, Reinhardt KR, Mayman DJ (2011) Radiographic analysis of a hand-held surgical navigation system for tibial resection in total knee arthroplasty. $\mathrm{J}$ Arthroplast 26(8):1527-1533. https://doi.org/10.1016/j.arth.2011. 01.012

37. Nam D, Nawabi DH, Cross MB, Heyse TJ, Mayman DJ (2012) Accelerometer-based computer navigation for performing the distal femoral resection in total knee arthroplasty. J Arthroplast 27(9):1717-1722. https://doi.org/10.1016/j.arth.2012.02.007

38. Nam D, Weeks KD, Reinhardt KR, Nawabi DH, Cross MB, Mayman DJ (2013) Accelerometer-based, portable navigation vs imageless, large-console computer-assisted navigation in total knee arthroplasty. A comparison of radiographic results. J Arthroplast 28(2):255-261. https://doi.org/10.1016/j.arth.2012.04.023

39. Nogler M, Kessler O, Prassl A, Donnelly B, Streicher R, Sledge JB, Krismer M (2004) Reduced variability of acetabular cup positioning with use of an imageless navigation system. Clin Orthop Relat Res 426:159-163

40. O’Donovan KJ, Kamnik R, O'Keeffe DT, Lyons GM (2007) An inertial and magnetic sensor based technique for joint angle measurement. J Biomech 40(12):2604-2611

41. Park FC, Martin BJ (1994) Robot sensor calibration: solving $\mathrm{AX}=\mathrm{XB}$ on the Euclidean group. IEEE Trans Robot Autom 10(5):717-721

42. Pflugi S, Liu L, Ecker TM, Schumann S, Larissa Cullmann J, Siebenrock K, Zheng G (2016) A cost-effective surgical navigation solution for periacetabular osteotomy (PAO) surgery. Int J Comput ssist Radiol Surg 11(2):271-280

43. Pflugi S, Vasireddy R, Liu L, Ecker TM, Lerch T, Siebenrock K, Zheng G (2016) A cost-effective navigation system for periacetabular osteotomy surgery. Int Conf Med Imaging Virtual Real 9805:84-95

44. Rambach JR (2016) Learning to fuse : a deep learning approach to visual-inertial camera pose estimation. In: IEEE international symposium on mixed and augmented reality (ISMAR), pp. 71-76

45. Rebello KJ (2004) Applications of MEMS in surgery. Proc IEEE 92(1):43-55 
46. Ren H, Kazanzides P (2014) Attitude tracking using an integrated inertial and optical navigation system for hand-held surgical instruments. In: International conference on control, automation and systems, pp. 290-293

47. Ryan JA, Jamali AA, Bargar WL (2010) Accuracy of computer navigation for acetabular component placement in THA. Clin Orthop Relat Res 468(1):169-177

48. Schweighofer G, Pinz A (2006) Robust pose estimation from a planar target. IEEE Trans Pattern Anal Mach Intell 28(12):20242030

49. Steppacher SD, Lerch TD, Gharanizadeh K, Liechti EF, Werlen SF, Puls M, Tannast M, Siebenrock KA (2014) Size and shape of the lunate surface in different types of pincer impingement: theoretical implications for surgical therapy. Osteoarthr Cartil 22(7):951-958

50. Steppacher SD, Tannast M, Ganz R, Siebenrock KA (2008) Mean 20-year followup of bernese periacetabular osteotomy. Clin Orthop Relat Res 466(7):1633-1644
51. Su S, Zhou Y, Wang Z, Chen H (2017) Monocular vision-and IMU-based system for prosthesis pose estimation during total hip replacement surgery. IEEE Trans Biomed Circuits Syst 11(3):1-10

52. Tibor LM, Sink EL (2012) Periacetabular osteotomy for hip preservation. Orthop Clin North Am 43(3):343-357

53. Walti J, Jost GF, Cattin PC (2014) A new cost-effective approach to pedicular screw placement. MICCAI AE-CAI - Lecture Notes in Computer Science 8678 (Lecture Notes in Computer Science), pp 90-97

54. Zhang H, Banovac F, Glossop N, Wood BJ, Lindisch D (2006) Electromagnetic tracking for abdominal interventions in computer aided surgery. Comput Aided Surg 11(3):127-136 